\title{
Projeto NECOS: Rumo ao Fatiamento Leve de Recursos em Infraestruturas de Nuvens Federadas
}

\author{
Fernando N. N. Farias ${ }^{1}$, Billy A. Pinheiro ${ }^{1}$, Antônio J. G. Abelém ${ }^{1}$, \\ Paulo D. Maciel Jr. ${ }^{2,5}$, André L. B. Rocha ${ }^{2}$, Fábio L. Verdi ${ }^{2}$, \\ Augusto V. Neto $^{6}$, Sand L. Correa ${ }^{4}$, Marcelo Nascimento ${ }^{3}$, \\ Rafael Pasquini $^{7}$, Christian Esteve Rothenberg ${ }^{8}$ \\ ${ }^{1}$ Universidade Federal do Pará (UFPA) \\ ${ }^{2}$ Universidade Federal de São Carlos (UFSCar) \\ ${ }^{3}$ Centro de Pesquisa e Desenvolvimento em Telecomunicações $(\mathrm{CPqD})$ \\ ${ }^{4}$ Universidade Federal de Goiás (UFG) \\ ${ }^{5}$ Instituto Federal da Paraíba (IFPB) \\ ${ }^{6}$ Universidade Federal do Rio Grande do Norte (UFRN) \\ ${ }^{7}$ Universidade Federal de Uberlândia (UFU) \\ ${ }^{8}$ Universidade Estadual de Campinas (UNICAMP) \\ \{fennf,billy,abelem\}@ufpa.br, \{beltrami,verdi\}@ufscar.br, marcelon@cpqd.com.br \\ sand@inf.ufg.br,paulo.maciel@ifpb.edu.br, augusto@dimap.ufrn.br, rafael.pasquini@ufu.br \\ chesteve@dca.fee.unicamp.br
}

\begin{abstract}
The Novel Enablers for Cloud Slicing (NECOS) project proposes a solution that seeks to automate the optimized cloud configuration process, providing uniform management with a high level of autonomy for the computing, connectivity and storage resources currently separated, based on the Lightweight Slice Defined Cloud (LSDC) concept. In this paper, we discuss the motivation, objectives, architecture, research challenges and initial efforts of the NECOS project through a set of use cases.
\end{abstract}

Resumo. O projeto Novel Enablers for Cloud Slicing (NECOS) propõe uma solução que visa automatizar o processo de configuração otimizada de nuvem e rede, fornecendo um gerenciamento uniforme com um alto nivel de autonomia para os recursos de computação, conectividade e armazenamento atualmente separados, baseado no conceito LSDC (Lightweight Slice Defined Cloud). Neste artigo, discute-se a motivação, objetivos, arquitetura, desafios de pesquisa e esforços iniciais do projeto NECOS através dos casos de uso definidos.

\section{Introdução}

A popularização das tecnologias de computação em nuvem (Cloud Computing - CC), tem incentivado o aumento no número de provedores que estão oferecendo serviços em qualquer um dos seus modelos XaaS, por exemplo, Software como Serviço (Software 
as a Service - SaaS), Plataforma como Serviço (Platform as a Service - PaaS), Rede como serviço ( Network as a Service - NaaS) e Infraestrutura como Serviço (do inglês Infrastructure as a Service - IaaS) [Mell and Grance 2011]. Diante desta variedade de serviços em nuvem, há um eminente desafio para que sejam fornecidos sob demanda respeitando os contratos de nível de serviço (Service Level Aggrement - SLA) utilizando recursos de computação, conectividade e armazenamento gerenciados separadamente.

Este desafio tem impulsionado a criação de várias soluções como o GENI ${ }^{1}$, que automaticamente agenda e provisiona recursos heterogêneos em rede, e o RESERVOIR ${ }^{2}$, que levou a orquestração de serviços [de Sousa et al. 2018] um pouco mais longe ao permitir a alocação e a implantação automática de recursos para os serviços à medida que são demandados. No entanto, estas soluções carecem de uma abstração única e escalável que permita atender as diferentes demandas dos serviços em um ambiente heterogêneo.

Para fornecer uma abstração única com abrangência fim-a-fim dos recursos das nuvens (processamento e armazenamento) e de redes, a plataforma $\mathrm{NECOS}^{3}$ utiliza o conceito de cloud network slicing [Silva et al. 2018]. Este conceito tem sido definido de diversas maneiras na literatura, mas em geral entende-se como uma composição de recursos (computação, rede e armazenamento) físicos e virtuais criada sob demanda, contando com uma infraestrutura subjacente comum, com controle, gerenciamento e orquestração independentes. Estas slices devem ser mutuamente isoladas e flexíveis o suficiente para acomodar diferentes necessidades dos inquilinos (tenants), fornecendo um modelo de Fatia como um Serviço (Slice as a Service) que provê ao tenant a gerência da infraestrutura virtual (Virtual Infrastructure Manager - VIM) sob demanda [Clayman et al. 2018].

Este artigo apresenta a versão atual da arquitetura do NECOS, os desafios identificados e os casos de uso refinados no decorrer do projeto e que hoje apresentam como principal diferencial a adição do cenário de serviços de IoT para cidades inteligentes.

\section{Proposta do NECOS}

Detalhes do projeto NECOS são descritos nesta seção, incluindo a abordagem LSDC (Lightweight Slice Defined Cloud), a arquitetura do NECOS, os principais desafios vinculados ao conceito de cloud network slices fim a fim (End-to-End - E2E), e por fim um workflow de sua criação.

\subsection{Lightweight Slice Defined Cloud (LSDC)}

A integração dos domínios de rede e computação em nuvem, como também suas operações de gerenciamento, irão permitir uma grande economia e a capacidade de ampliar a flexibilidade do provisionamento de serviços nestas áreas. Atualmente, essas operações são realizadas separadamente e, portanto, combiná-las é uma meta do NECOS com o objetivo de simplificar suas ações. Ao fazer isso, o Projeto NECOS alavancará o amadurecimento dos paradigmas SDN (Software Defined Networking) [Kreutz et al. 2015] e virtualização de rede que, através de um projeto e implementações adequadas, ajudará a cumprir os requisitos de inovação de maneira mais confiável, rápida e simples [Manzalini et al. 2016].

\footnotetext{
${ }^{1}$ http://www.geni.net/

${ }^{2}$ https://www.reservoir-fp7.eu

${ }^{3}$ http://www.h2020-necos.eu/
} 
Na visão do NECOS, o conceito de cloud network slicing baseia-se em recursos e funções já disponíveis na plataforma de nuvem. A slicing é uma técnica associada ao particionamento de recursos, capaz de criar e redefinir partições conforme necessário. Um slice pode ser entendido como um agrupamento de recursos físicos ou virtuais que podem atuar como sub-nuvem e sub-rede aparentemente independentes, podendo acomodar componentes do serviço. As principais características da cloud network slicing incluem:

- A implantação simultânea de várias slices lógicas, auto-suficientes e independentes, compartilhadas ou particionadas em uma infraestrutura comum.

- Suporte a multi-serviço dinâmico, multi tenancy e o meio de integração entre os players do mercado.

- A separação de funções simplifica o provisionamento de serviços, a gerência de redes e os desafios relacionados à operacionalidade e integração, especialmente para suportar os serviços de comunicação.

- Operadores de rede, provedores de serviço Internet e proprietários de infraestrutura de nuvem podem explorar a tecnologia slicing para: reduzir os gastos operacionais, permitir a programabilidade e a inovação necessárias para enriquecer os serviços oferecidos, disponibilizar serviços personalizados e permitir a capacidade de programabilidade da rede para provedores OTT (Over-The-Top ou content providers) e outros players do mercado sem alterar a infraestrutura física.

O LSDC representa uma nova abordagem para automatizar o processo de configuração otimizada de nuvens pela criação do conceito de cloud network slicing em todos os recursos em conjunto de centros de dados federados, bem como, fornecer um gerenciamento uniforme dos recursos de computação, conectividade e armazenamento.

\subsection{Arquitetura do NECOS}

A arquitetura completa da plataforma NECOS, apresentada na Figura 1, define os componentes para o aprovisionamento de cloud network slices E2E em uma federação multitecnológica e de múltiplos provedores. No NECOS, um cloud network slice E2E pode ser composto por múltiplas partes (cloud slices) aprovisionadas em diferentes centros de dados da federação, e são interconectadas por network slices.

Para facilitar a compreensão da arquitetura do NECOS, inicialmente apresentada à comunidade no Entregável D3.1 do NECOS [NECOS 2018b], nós definimos, na Figura 1, quatro áreas distintas, isto é, a do Tenant em vermelho, a do Provedor de Recursos em verde, a do Marketplace em amarelo e a do Provedor de Slice em azul. A seguir, faremos uma descrição dos principais componentes da arquitetura que merecem ser destacados: o Slice Orchestrator, o componente de gerência e monitoramento IMA e o Marketplace.

O Slice Orchestrator realiza manipulação de slices, isto é, requisita ao Marketplace as diferentes partes que serão incluídas em uma slice E2E através da realização de uma fase de orquestração inicial. Adicionalmente, ele supervisiona o gerenciamento do ciclo de vida dos serviços que estão sendo executados nas slices, baseado nas informações coletadas dos diferentes recursos presentes na slice pelo componente IMA.

O componente IMA (do inglês, Infrastructure \& Monitoring Abstraction) oferece uma interface northbound abstrata que permite ao Slice Orchestrator executar suas funções enquanto as informações das partes das slices permanecem transparentes. Como 


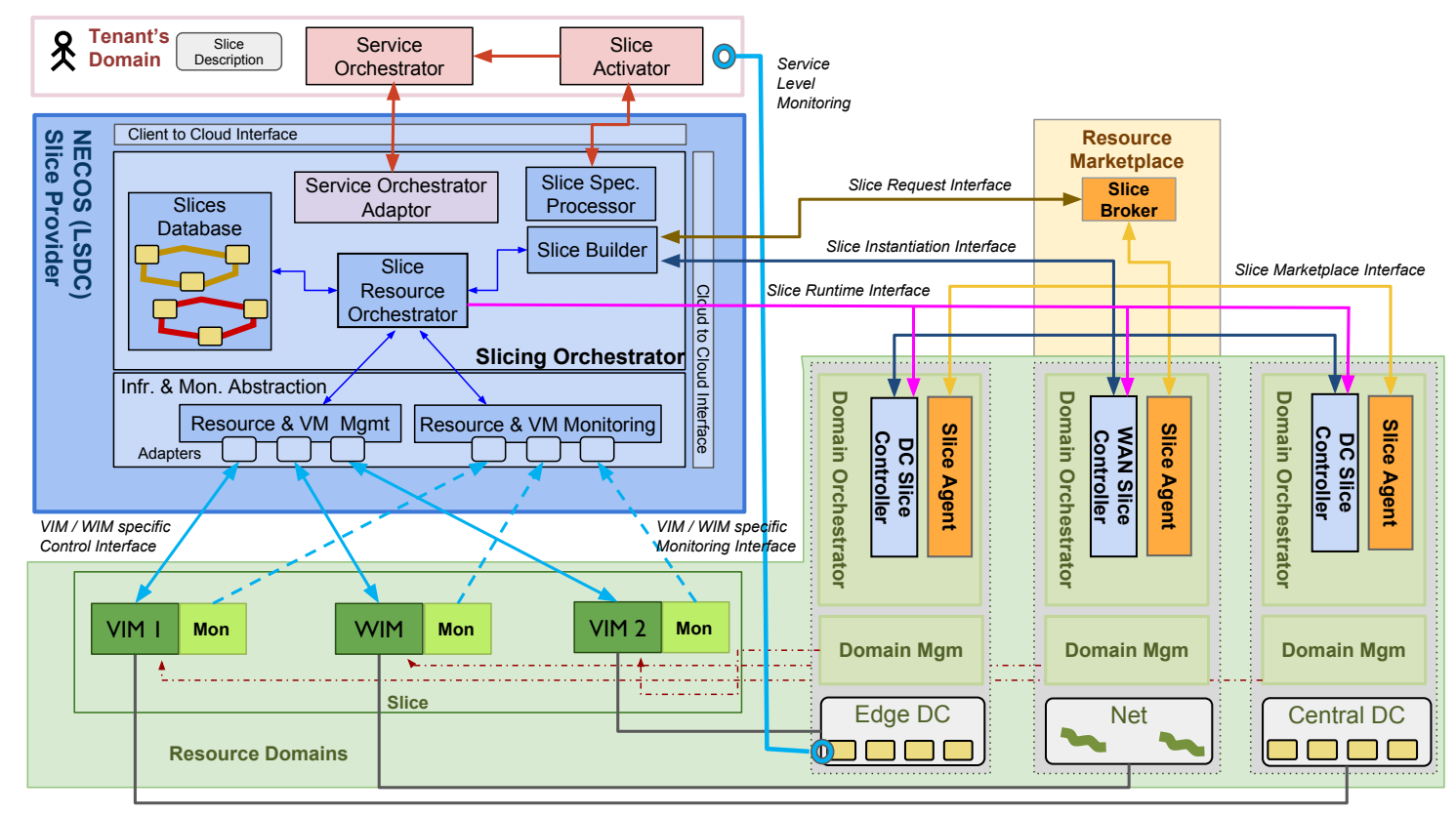

Figura 1. Arquitetura NECOS. Fonte e mais informações: NECOS D3.1.

exemplo de tais funções podemos citar: a coleta de informações sobre topologia de recursos e informações de monitoramento de recursos para cada parte da slice; o monitoramento e a verificação do estado dos elementos virtuais alocados a cada parte da slice; a coleta de indicadores KPIs (do inglês, Key performace Indicators) voltados para recursos como CPU, memória e armazenamento. Para alcançar estes objetivos, múltiplos adaptadores traduzem as requisições provenientes da interface northbound para o VIM ou WIM (do inglês, Virtual/WAN Infrastracture Manager). Deste modo, eles abstraem as partes das slices heterogeneamente na interface southbound do IMA.

A descoberta de recursos que irão compor uma slice E2E é responsabilidade do componente Marketplace, que é um sistema distribuído responsável por localizar partes de slices adequadas a partir de um conjunto de infraestruturas de nuvem federadas. Como mostrado na Figura 1, o Marketplace é formado por dois componentes, nomeados de Slice Broker e Slice Agent. O Slice Broker descobre as partes das slices através da interação com um conjunto de Slice Agents hospedados nos domínios de recursos envolvidos.

\subsection{Desafios do Projeto}

Embora a abordagem de prover slice como serviço proposta pelo NECOS traga inúmeros benefícios para tecnologias emergentes, como por exemplo, a computação em nuvem, redes móveis 5G, diversas verticais de IoT (do inglês, Internet of Things), entre outras, há ainda algumas lacunas e desafios que necessitam atenção para suprir eficientemente as necessidades que tangenciam este novo conceito denominado slicing. Alguns dos principais desafios vislumbrados são descritos a seguir.

Virtualização da Infraestrutura Física: Este conceito já vem sendo discutido há um certo tempo, entretanto com o surgimento do conceito slicing, aspectos importantes precisam ser melhorados, como por exemplo, o isolamento entre os recursos, a escalabilidade e o desempenho. 
Elasticidade Computacional: A elasticidade de uma slice é de extrema importância para que o uso dos recursos seja otimizado de modo a respeitar as SLAs acordadas. Tal elasticidade pode ocorrer no nível de infraestrutura física, virtual ou até mesmo no nível da aplicação.

Heterogeneidade: A heterogeneidade inserida pelos diferentes provedores de infraestrutura é um desafio para ser estudado. Sendo assim, cabe ao Slice Provider abstrair as diferentes tecnologias e infraestruturas dos provedores, de modo a entregar uma visão transparente e unificada dos recursos E2E que o Tenant requisitou.

Segurança: A segurança é um dos desafios tradicionais no escopo de redes de computadores em geral. Sendo assim, para o conceito de slicing que envolve diferentes infraestruturas, e a comunicação entre diferentes provedores, os desafios são ainda maiores.

Gerência da Mobilidade: A mobilidade de dispositivos que necessitam de constante conexão é uma característica relacionada a diversos casos de uso envolvendo slices. Sendo assim, a gerência, a melhoria do tráfego e a melhoria dos protocolos de comunicação dos dispositivos móveis são pontos que devem ser aprimorados.

\subsection{Workflow de Criação de Slices}

Esta Seção apresenta um workflow, usando a metodologia BPMN (do inglês, Business Process Model and Notation), que relaciona os componentes presentes na arquitetura proposta na Figura 1. O workflow descrito, que é detalhado no Entregável D5.1 do NECOS [NECOS 2018c], envolve a operação de criação de uma nova slice.

O workflow apresentado na Figura 2 adota a seguinte notação: o círculo em verde denota o início enquanto o laranja denota o término do workflow. Os retângulos cinzas representam os componentes da arquitetura do NECOS. Os retângulos tracejados, que agrupam componentes, correspondem as áreas descritas na Seção 2.2. Por fim, as interfaces de comunicação entre os componentes são ilustradas pelos círculos em azul.

A Figura 2 ilustra todos os módulos da arquitetura NECOS envolvidos no workflow de Criação da Slice, as flechas representam as interações entre os componentes para

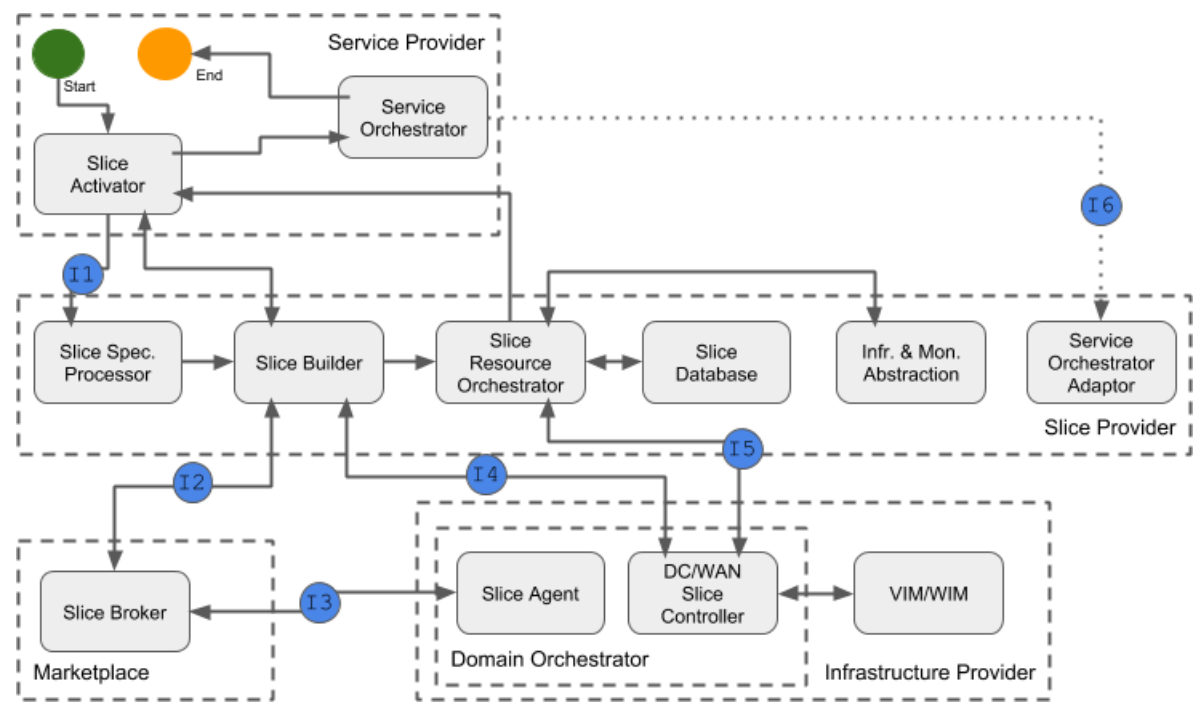

Figura 2. Workflow de Criação da Slice Fim-a-Fim. Fonte e mais informações: NECOS D5.1. 
a criação da slice. O workflow é iniciado pelo tenant no Service Provider através do componente Slice Activator, sua função é iniciar a requisição desta nova slice para o Slice Provider interagindo com o componente Slice Specification Processor via Interface 1 (I1). O Slice Specification Processor irá extrair as especificações de infraestrutura e serviço requisitadas pelo Tenant e então envia para o Slice Builder. O Slice Builder em conjunto com os componentes Slice Broker (via I2) e Slice Agent (via I3) possui como função analisar os recursos disponíveis nos provedores de infraestrutura e então otimizar a alocação dos recursos ofertados pelo Marketplace. Após definir quais provedores de infraestrutura irão compor a slice, o Slice Builder se comunica com o DC/WAN Slice Controller de cada um dos provedores para instanciar os recursos que foram requisitados pelo $\mathrm{Te}$ nant (via I4). O DC WAN Slice Controller retorna para o Slice Builder os apontadores para os novos recursos, e então a informação é enviada para o Slice Resource Orchestrator que possui como responsabilidade orquestrar os recursos instanciados de modo a otimizar a utilização dos recursos presentes na slice. O Slice Resource Orquestrator é um componente de suma importância na arquitetura do NECOS, pois ele além de ser responsável pela orquestração dos recursos, ele também é responsável pela instanciação dos VIMs/WIMs. A instanciação destes elementos é de suma importância para o deployment de um novo serviço e/ou recurso virtual (Máquina Virtual, Container) para isto ele utiliza a Interface 5 (I5) na qual ele se comunica diretamente com os DC/WAN Slice Controllers. Além desta função, o Slice Resource Orchestrator gerencia o componente de monitoramento da slice (IMA) assim como o banco de dados que irá armazenar tanto as informações da slice quanto às KPIs monitoradas pela IMA. Por fim, após a slice estar operante, os serviços do Tenant podem ser instanciados e então orquestrados pelo Service Orchestrator via a Interface 6 (I6) na parte mais à direita da figura.

\section{Casos de Uso}

O projeto NECOS identificou Telco Cloud e o MEC (do inglês, Multi-access Edge Computing) como casos de uso para definir uma arquitetura de federação de nuvem que suporta slicing como um serviço. Esses dois casos de uso, apresentados na Figura 3, estão atraindo bastante atenção na indústria com o desenvolvimento de padrões relacionados a eles e o aumento das ofertas comerciais.

Do ponto de vista da Telco Cloud, várias iniciativas estão progredindo, como o CORD no ONF e a abordagem CloudCO no BBF, conforme relatado no Entregável D2.2 [NECOS 2018a]. As arquiteturas ali definidas continuam a evoluir e espera-se que possam ser incorporadas em ofertas comerciais no curto prazo. Da mesma forma, o ambiente de computação de borda está se expandindo além do ETSI MEC, multiplicando o número de soluções, tais como o EdgeX Foundry, o grupo de trabalho TIP do Facebook em Edge Computing, e Akraino, para mencionar algumas.

Todas essas iniciativas reforçam o escopo do NECOS como uma solução crossover de fatiamento que poderia ser implantada para federar opções tão diversas, tanto na Telco Cloud quanto no MEC. Além dos casos de uso, o NECOS definiu alguns cenários de aplicabilidade que podem usar esse tipo de ambiente de execução. Os cenários permitiram que a noção de serviço seja executada na slice fornecida pelo sistema.

Dentro do contexto destes dois casos de uso, alguns cenários foram criados para obter os requisitos iniciais da plataforma NECOS e para mostrar sua aplicação em dife- 


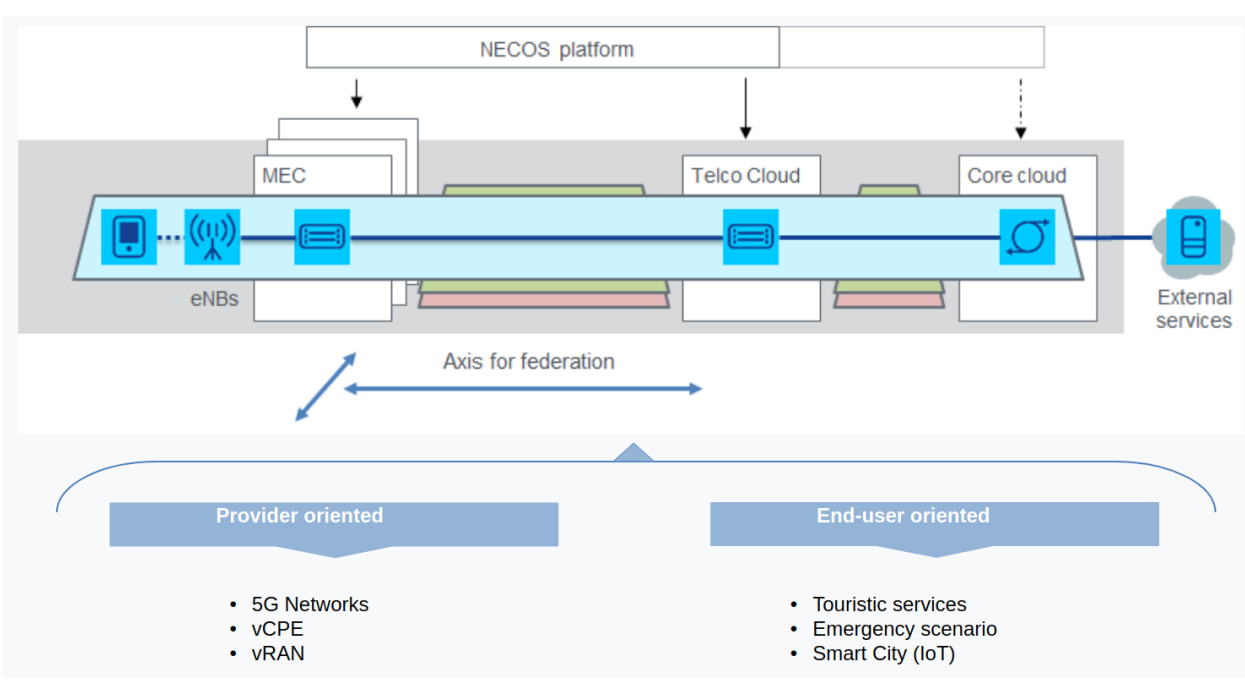

Figura 3. Casos de Uso. Fonte e mais informações: NECOS D2.2.

rentes contextos de rede. A seguir, sumarizamos estes cenários e suas derivações.

vRAN: do inglês, virtual Radio Access Network, o cenário vRAN aproveita as tendências de virtualização para fornecer funções de rede associadas à RAN e também pode ser estendido para suportar diferentes provedores de serviços.

Serviços 5G: este cenário aborda os requisitos das operadoras de rede para adaptar as redes existentes para fornecer serviços 5G futuros, como o Banda Larga Móvel aprimorado (eMBB), o compartilhamento de vídeo de alta definição, as comunicações maciças de tipo de máquina (mMTC) e as comunicações ultraconfiáveis e de baixa latência (uRLLC).

vCPE: do inglês, virtual Customer Premises Equipment, esse cenário explora os ambientes de Telecomunicações voltados para o uso de recursos de nuvem, o que permite a evolução dos Central Offices, aplicando a integração de elementos virtuais e não-virtuais. Distribuição de Conteúdo Turístico: este cenário aborda a necessidade de um Centro Metropolitano de Turismo para fornecer conteúdo turístico com reconhecimento de local a um grande número de visitantes em uma área metropolitana.

Próxima Geração de Aplicações Turísticas: espera-se que os aplicativos de realidade aumentada melhorem a experiência dos viajantes e aumentem o valor do produto cultural oferecido pelas autoridades municipais.

Distribuição de Conteúdo em Cidades Inteligentes: este cenário aborda a necessidade de integrar diferentes provedores de infraestrutura em uma cidade para fornecer a entrega de conteúdo de dados em uma Cidade Inteligente. Um contribuição inicial está descrita em [Carmo et al. 2019], que propõe uma abordagem para definir em slices as infraestruturas de redes de acesso WiFi compartilhadas.

Monitoramento Metropolitano Integrado: este cenário aborda um Centro de Segurança Pública responsável por melhorar o gerenciamento e resolução de incidentes para um número significativo de socorristas (por exemplo, departamento de polícia, serviços de incêndio e resgate, serviços médicos de emergência e obras), bem como sua interação com a população civil em uma área metropolitana. 


\section{Conclusões}

Neste artigo, apresenta-se a proposta NECOS que objetiva abordar as limitações das infraestruturas atuais de nuvem, propondo a abordagem Lightweight Slice Defined Cloud (LSDC). O LSDC atua como ativador ao Cloud Slicing por meio do conceito de Slice as a Service. Além disso, ele oferece uma estrutura de gerenciamento unificada para domínios tanto de rede quanto computacionais, e também, fornece uma orquestração inteligente para infraestruturas de nuvem federadas.

\section{Agradecimentos}

Esta pesquisa é financiada pela Comissão Europeia e Ministério da Ciência, Tecnologia, Inovação e Comunicação (MCTIC) através da RNP e CTIC no contexto da $4^{\circ}$ chamada conjunta EU-BR, acordo \#777067 (NECOS - Novel Enablers for Cloud Slicing). Este estudo foi financiado em parte pela CAPES - Código Financeiro 001, e pelo CNPq.

\section{Referências}

Carmo, M., Silva, F. S. D., Neto, A. V., Corujo, D., and Aguiar, R. (2019). Network-cloud slicing definitions for wi-fi sharing systems to enhance $5 \mathrm{~g}$ ultra dense network capabilities. Wireless Communications and Mobile Computing, 2019.

Clayman, S., Tusa, F., and Galis, A. (2018). Extending Slices into Data Centers: the VIM ondemand model. In 2018 9th International Conference on the Network of the Future (NOF), pages $31-38$.

de Sousa, N. F. S., Perez, D. A. L., Rosa, R. V., Santos, M. A. S., and Rothenberg, C. E. (2018). Network Service Orchestration: A Survey. http://arxiv.org/abs/1803.06596.

Kreutz, D., Ramos, F. M. V., Veríssimo, P. E., Rothenberg, C. E., Azodolmolky, S., and Uhlig, S. (2015). Software-Defined Networking: A Comprehensive Survey. Proceedings of the IEEE.

Manzalini, A., Buyukkoc, C., CHEMOUIL, P., Kuklinski, S., Callegati, F., Galis, A., Odini, M.P., I, C.-L., Huang, J., Bursell, M., Crespi, N., Healy, E., and Sharrock, S. (2016). Towards 5G Software-Defined Ecosystems: Technical Challenges, Business Sustainability and Policy Issues. Research report.

Mell, P. M. and Grance, T. (2011). Sp 800-145. the nist definition of cloud computing. Technical report, Gaithersburg, MD, United States.

NECOS (2018a). D2.2: Consolidated definition of use cases, business models and requirements analysis. http://www.maps.upc.edu/public/d2.2_final_v2.0.pdf.

NECOS (2018b). D3.1: Necos system architecture and platform specification. http://www . maps.upc.edu/public/NECOS20D3.120final.pdf.

NECOS (2018c). D5.1: Architectural update, monitoring and control policies frameworks. http://www.maps.upc.edu/public/D5.1_final.pdf.

Silva, F. S. D., Lemos, M. O. O., Medeiros, A., Neto, A. V., Pasquini, R., Moura, D., Rothenberg, C., Mamatas, L., Correa, S. L., Cardoso, K. V., Marcondes, C., ABelem, A., Nascimento, M., Galis, A., Contreras, L., Serrat, J., and Papadimitriou, P. (2018). Necos project: Towards lightweight slicing of cloud federated infrastructures. In 2018 4th IEEE Conference on Network Softwarization and Workshops (NetSoft), pages 406-414. 